\title{
Real World Experience of Drug Induced Liver Injury in Patients Undergoing Chemotherapy
}

\author{
Adiba Azad ${ }^{1}$, Paul Chang ${ }^{1}$, Deepika Devuni ${ }^{1}$, Kian Bichoupan ${ }^{1}$, Varun Kesar ${ }^{2}$, Andrea D. Branch ${ }^{1}$, \\ William K. Oh ${ }^{1}$, Matthew D. Galsky ${ }^{1}$, Jawad Ahmad ${ }^{1}$ and Joseph A. Odin 1,* \\ ${ }^{1}$ Icahn School of Medicine, Mount Sinai, New York, NY, USA \\ ${ }^{2}$ Lenox Hill Hospital, New York, NY, USA
}

${ }^{*}$ Corresponding author: Joseph A. Odin, Icahn School of Medicine, Mount Sinai, New York, NY, USA, Tel: 212-241-0034; E-mail: joseph.odin@mountsinai.org

Rec date: July 31, 2018; Acc date: September 10, 2018; Pub date: September 14, 2018

Citation: Azad A, Chang P, Devuni D, Bichoupan K, Kesar V, et al. (2018) Real World Experience of Drug Induced Liver Injury in Patients Undergoing Chemotherapy. J Clin Gastroenterol Hepatol Vol.2: No.3:18.

\section{Abstract}

Background \& Aim: To better understand the clinical significance of drug induced liver injury (DILI) during chemotherapy, we examined the epidemiology, incidence, and treatment effects of DILI in patients undergoing chemotherapy for genitourinary malignancies over a twoyear period.

Methods: We conducted a retrospective review of 284 patients who underwent chemotherapy for prostate, bladder, testicular and renal cell carcinomas over a two year period. Those with abnormal or absent liver test (LT) results prior to chemotherapy initiation were excluded. Post chemotherapy LT results were defined as DILI if ALT>3x ULN and/or total bilirubin (TB) $>2 x$ ULN, in the absence of other more likely causes of elevated LT.

Results: The cumulative incidence of DILI in the total study population was $6.1 \%(17 / 284)$, and in the population who had appropriate LT performed it increased to $18.9 \%(17 / 90)$. Chemotherapeutic agents were determined to be the cause of DILI in $82 \%(14 / 17)$ of patients, and the treatment plans were changed in $59 \%$ (10/17) of patients.

Conclusion: In this real world study, the cumulative incidence of DILI was higher than commonly reported in clinical trials, and the majority of affected patients had to have their cancer treatment altered or interrupted.

Keywords: Drug induced liver injury; Chemotherapy; Genitourinary malignancies; Liver tests

\section{Introduction}

Drug induced liver injury (DILI) represents an insult to the liver by various compounds ranging from medications to herbal supplements. Though not exceedingly common, DILI represents a significant concern in medical practice. Medications cause $85 \%$ of DILI cases, whereas herbal and dietary supplements account for the remaining 15\% [1]. Acetaminophen with its dose-dependent hepatotoxicity is the most common drug implicated in DILI, followed by antibiotics of which amoxicillin-clavulanate is the leading culprit of idiosyncratic DILI [2]. DILI frequently results in treatment changes, drug withdrawal from the market [3], and has been shown to cause $11 \%$ of the acute liver failure cases in the United States [4].

Pharmacologically, DILI can occur as a result of a predictable, dose-dependent process, or an idiosyncratic, unpredictable dose-independent mechanism. Biochemically, the injury can be hepatocellular resulting in an elevation in serum transaminases, cholestatic with an elevation in the bilirubin levels, or a mixture of both, which do not correlate strongly with changes in liver histology [5].

Chemotherapeutic agents are relatively rarely reported causes of DILI, but they are associated with significant morbidity and mortality. The frequency of chemotherapy induced liver injury may be under reported because of the challenges of making a diagnosis in oncology patients due to the presence of other potentially hepatotoxic medications, high rates of infections, radiation therapy, and metastatic involvement of the liver [6]. Additionally, these patients are typically closely monitored, and chemotherapy is often halted before liver biochemistries increase significantly.

Treatment of genitourinary (GU) malignancies frequently includes agents associated with DILI. However, there is rudimentary information on the frequency of DILI and the causative agents in this particular patient population. Pazopanib, a tyrosine kinase inhibitor with multiple targets, is most well studied in GU cancer patients, with reported rates of elevated alanine transaminase elevation $(A L T)>3 x$ upper limit of normal (ULN) of $20 \%$, as shown in a meta-analysis of 8 clinical trials of the drug [7]. However, the incidence and characterization of other chemotherapeutic agents, such as the taxanes, have not been previously studied in GU cancer patients. 
The purpose of this study was to examine the cumulative incidence of DILI in patients being treated for GU malignancies and its effect on cancer treatment.

\section{Methods}

\section{Study design and data source}

This study was a retrospective review using a prospectively collected database of patients undergoing chemotherapy for GU malignancies in a large tertiary care center, from January 2013 to December 2014. The database contains both inpatient and outpatient medication records, and demographic data. Initial and follow up LT were performed per protocol, not for suspected liver injury.

\section{Inclusion/exclusion criteria}

Eligible patients were on chemotherapy with documented dates for treatment initiation and termination and had normal LT results within 4 weeks prior to initiating treatment. Patients were excluded if they had chronic liver disease, progression of liver metastases following chemotherapy, less than two LT drawn from start to end of treatment, and abnormal or absent LT prior to drug therapy.

\section{DILI definitions}

Elevated liver chemistries were defined as DILI if they met the above criteria, had ALT $>3 x$ ULN and/or total bilirubin (TB) $>2 x$ ULN, and other more likely causes of elevated LT were ruled out by expert hepatologist opinion (authors JAO, JA). Liver injury was defined as hepatocellular if $A L T>3 x$ ULN and $T B<2 x$ ULN, cholestatic if $A L T<3 x$ ULN and $T B>2 x$ ULN, and mixed if $A L T>3 x$ ULN and TB>2x ULN after start of treatment.

\section{DILI severity}

Two scoring systems were used to address DILI severity: a) a five point scale developed by the US DILI Network (US DILIN) for grading the severity of liver injury based on the presence of jaundice, coagulopathy, hospitalization, signs of other organ failure, and ultimate outcome and b) a grading scheme published by the National Cancer Institute $(\mathrm{NCl})$ called the Common Terminology Criteria for Adverse Events (CTCAE) $[8,9]$. The CTCAE comprises standardized definitions of adverse events defined as abnormal clinical findings temporally associated with the use of a therapy, and ranges from Grade 1 (mild) to Grade 4 (life threatening), with organ specific parameters involved, including the hepatobiliary system.

\section{Outcome}

The primary outcome we studied was the cumulative incidence of DILI in patients undergoing chemotherapy.

\section{Statistical analyses}

SPSS software (SPSS Inc., Chicago, IL) was used to perform statistical analyses. Parameters are described as mean \pm SD or percentage where appropriate. Chi-square test was used to compare categorical variables and student t-test was used to analyze continuous variables. Logistic regression analysis was performed to show the significance, if any, of associated factors with DILI. Odds ratios (OR) and their $95 \%$ confidence intervals $(\mathrm{Cl})$, were calculated for the logistic regression model. $P$ values $\leq 0.05$ were considered statistically significant.

\section{Results}

A total of 284 patients with prostate, bladder, testicular and renal cell carcinomas received chemotherapy from January $1^{\text {st }}$ 2013 to December $31^{\text {st }} 2014$ at the Mount Sinai Hospital. Patients who did not have normal LT results available within three months (baseline LT results) prior to the initiation of chemotherapy, and abnormal baseline LT results were excluded ( $\mathrm{N}=194)$, leaving 90 cases (Figure 1).

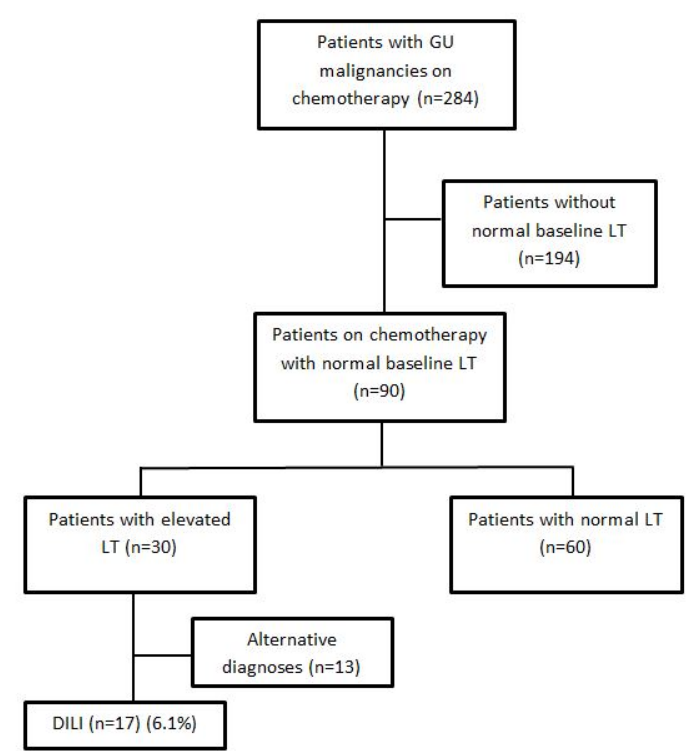

Figure 1: Patient flow chart. The overall incidence of DILI during chemotherapy was $6.1 \%(17 / 284)$. If those without known normal baseline LT results are excluded, the incidence of DILI was $18.9 \%$ (17/90). The cause of elevated LT results during chemotherapy most often was DILI (17/30).

Sixty patients had normal serial LT results after initiation of drug therapy and were identified as control cases. Thirty patients had elevated LT results that met DILI criteria after initiation of drug therapy. Out of these 30 patients who had elevated LT results, 13 had likely alternative etiologies for their abnormal liver chemistries (Table 1), and the remaining were identified as probable DILI cases ( $N=17)$. The incidence of DILI in the overall cohort was $6.1 \%(17 / 284)$. Of the patients who had baseline and follow up LT performed the incidence of DILI 
rose to $18.9 \%(17 / 90)$. The cause of elevated LT results during chemotherapy most often was DILI (17/30).

Table 1: Etiologies for abnormal LT results based on medical record review.

\begin{tabular}{|l|l|}
\hline Etiology & $\mathbf{N = 1 3}(\%)$ \\
\hline Metastases & $5(38)$ \\
\hline Multi organ failure/shock & $4(31)$ \\
\hline Biliary tree obstruction & $2(15)$ \\
\hline NSTEMI & $1(8)$ \\
\hline Chronic liver disease & $1(8)$ \\
\hline
\end{tabular}

The demographic data and baseline clinical data of the control $(N=60)$ and the DILI cases $(N=17)$ are presented in Table 2.

Table 2: Cancer type, demographic and baseline clinical data comparison between control and DILI cases.

\begin{tabular}{|c|c|c|c|}
\hline Variables & $\begin{array}{l}\text { Control } \\
n=60\end{array}$ & $\begin{array}{l}\text { DILI } n= \\
17\end{array}$ & $\begin{array}{l}\mathrm{p}- \\
\text { value }\end{array}$ \\
\hline \multicolumn{4}{|l|}{ Cancer type (\%) } \\
\hline Prostate & $32(53)$ & $6(35)$ & \multirow{4}{*}{0.06} \\
\hline Bladder & $12(20)$ & $3(18)$ & \\
\hline Renal cell & $15(25)$ & $5(29)$ & \\
\hline Testicular & $1(2)$ & $3(18)$ & \\
\hline \multicolumn{4}{|l|}{ Sex (\%) } \\
\hline Male & $53(88)$ & $14(82)$ & 0.68 \\
\hline \multicolumn{4}{|l|}{ Race (\%) } \\
\hline White & $28(47)$ & $6(35)$ & \multirow{4}{*}{0.59} \\
\hline Black & $13(22)$ & $3(18)$ & \\
\hline Asian & $1(2)$ & $0(0)$ & \\
\hline Unknown & $18(30)$ & $8(47)$ & \\
\hline \multicolumn{4}{|l|}{ Ethnic Group (\%) } \\
\hline Non-Hispanic & $40(67)$ & $9(53)$ & \multirow{3}{*}{0.29} \\
\hline Hispanic & $8(13)$ & $5(29)$ & \\
\hline Unknown & $12(20.0)$ & $3(17.6)$ & \\
\hline Age, yr, mean (SD) & $65.3(13.5)$ & $\begin{array}{l}64.9 \\
(18.7)\end{array}$ & 0.91 \\
\hline BMI, mean (SD) & $27.9(5.4)$ & $25.5(5.5)$ & 0.12 \\
\hline eGFR, ml/min/1.73 m2, mean (SD) & $54.0(11.3)$ & $56.6(8.4)$ & 0.4 \\
\hline Albumin, g/dl, mean (SD) & $4.1(0.4)$ & $3.7(0.6)$ & 0.007 \\
\hline
\end{tabular}

Overall, the most prevalent was prostate cancer in both control and cases, followed by renal cell carcinoma and bladder cancer. Testicular cancer was the least common in both groups. There was a trend towards a higher percentage of testicular cancer cases among those with DILI compared to the control group. Males were over-represented in both groups due to the higher prevalence of prostate cancer compared to the other GU malignancies.

The mean pre-treatment body mass index (BMI), age, and renal function did not show a difference between the two groups. Pre-treatment albumin in the DILI group $(3.7 \mathrm{~g} / \mathrm{dl})$ was significantly lower than in the control group $(4.1 \mathrm{~g} / \mathrm{dl}), \mathrm{p}=0.007$ (Table 2). Other pre-treatment clinical factors such as age, race, gender, BMI and eGFR were comparable between the DILI cases and control. The largest group receiving a specific drug comprised those receiving pazopanib. Similar to the overall DILI cases, in patients taking pazopanib, we found a trend towards a lower pre-treatment albumin in the DILI cases when compared to the control $(3.6 \mathrm{~g} / \mathrm{dl}$ vs. $3.9 \mathrm{~g} / \mathrm{dl}), \mathrm{p}=0.068$. Upon logistic regression analysis, of the factors included, only albumin showed a significant inverse correlation with DILI (Table 3).

Table 3: Logistic regression analysis of factors associated with DILI.

\begin{tabular}{|c|c|c|c|}
\hline Variables & Odds Ratio & $95 \%$ confidence interval & p-value* \\
\hline Age & 1.03 & $0.96-1.10$ & 0.38 \\
\hline Gender & 0.65 & $0.08-5.57$ & 0.69 \\
\hline \multicolumn{4}{|l|}{ Race } \\
\hline White & 1.15 & $0.03-8.61$ & 0.65 \\
\hline Black & 0.69 & $0.04-11.56$ & 0.8 \\
\hline Asian & 0 & 0 & 1 \\
\hline \multicolumn{4}{|l|}{ Ethnic group } \\
\hline Non-Hispanic & 1.79 & $0.14-23.00$ & 0.66 \\
\hline Hispanic & 1.59 & $0.12-21.94$ & 0.73 \\
\hline \multicolumn{4}{|l|}{ Cancer type } \\
\hline Prostate & 0.024 & $0.001-1.05$ & 0.05 \\
\hline Bladder & 0.026 & $0.001-0.98$ & 0.05 \\
\hline Renal & 0.032 & $0.001-1.17$ & 0.06 \\
\hline eGFR & 1.02 & $0.94-1.09$ & 0.69 \\
\hline Albumin & 0.17 & $0.035-0.79$ & 0.02 \\
\hline \multicolumn{4}{|c|}{$\begin{array}{l}\text { *Male was used as a reference to compare gender, cancer type was } \\
\text { compared against testicular, and race and ethnicity were compared against } \\
\text { the unknown groups. }\end{array}$} \\
\hline
\end{tabular}

Upon a thorough chart review of all cases, DILI resulted in changes in the treatment plan in $59 \%$ of the cases (10/17). The drug was discontinued in 7 cases, and the dose was reduced or administration delayed in 3 cases. Nine out of the aforementioned ten cases showed normalization of the LT results after drug was discontinued or the regimen changed, leaving one patient who had a fatal cardiac arrest before LT results normalized. Mortality data was collected until May 2016 with a median follow-up of 8 months, and showed no statistically significant difference between the control and DILI group, $18 \%$ (11 of 60 ) and $29 \%$ (5 of 17) respectively. 
The DILI cases were evenly divided between hepatocellular $(\mathrm{N}=9)$ and cholestatic DILI $(\mathrm{N}=8)$. Mean DILI latency for all the cases was 30 days, range 4-89 (Table 4). In general, hepatocellular DILI developed after a shorter time period than cholestatic DILI, $24 \pm 19$ vs. $40 \pm 32$ days respectively.

Table 4: DILI latency represented in days.

\begin{tabular}{|l|l|l|l|l|}
\hline \multirow{2}{*}{ Variables } & Time to DILI (all cases) & Time to DILI (Hepatocellular) & Time to DILI (Cholestatic) & Time to DILI (Pazopanib) \\
\cline { 2 - 5 } & $\mathbf{n = 1 7}$ & $\mathbf{n = 9}$ & $\mathbf{n = 8}$ & $\mathbf{n = 4}$ \\
\hline Mean \pm SD & $30 \pm 26$ & $24 \pm 19$ & $40 \pm 32$ & $50 \pm 28$ \\
\hline Median (range) & $27(4-89)$ & $17(4-50)$ & $30(7-89)$ & $42(27-89)$ \\
\hline
\end{tabular}

Chemotherapy agents were adjudicated to be the implicated agents in $82 \%(\mathrm{~N}=14)$ of the DILI cases, with the remaining three cases probably due to a statin, cyclooxygenase-2 inhibitor, and anti-retroviral agent taken during chemotherapy. The frequency of DILI associated with individual chemotherapeutic regimens and drug classes varied widely (Table 5). Individual drugs or regimens tended to be more associated with either hepatocellular or cholestatic DILI. DILI severity was assessed using distinct scoring systems developed by the US DILIN and NCI (CTCAE). According to the US DILIN grading scale, the DILI episodes were mild to moderate, and according to the CTCAE scale the episodes mostly were moderate to severe (Table 5).

Table 5: Characterization of DILI frequency, type, and severity by chemotherapy drug category.

\begin{tabular}{|c|c|c|c|c|c|c|c|}
\hline Drug Category & \#Cases & \#Control & $\begin{array}{l}\text { DILI frequency } \\
(\%)\end{array}$ & $\begin{array}{lll}\% & H C & \text { DILI } \\
(\mathrm{HC} / \mathrm{C}) & \end{array}$ & $\begin{array}{l}\text { DILI Severity Index } \\
\text { (range) }\end{array}$ & $\begin{array}{l}\mathrm{NCI} \text { CTCAE Liver } \\
\text { (range) }\end{array}$ & Toxicity \\
\hline $\begin{array}{l}\text { Paclitaxel } \\
\text { Carboplatin }\end{array}$ & 2 & 1 & 67 & $100(2 / 0)$ & Moderate & $2-4$ & \\
\hline Taxane group & 5 & 5 & 50 & $80(4 / 1)$ & Mild-Moderate & $2-4$ & \\
\hline Platinum derivatives & 5 & 8 & 38 & $80(4 / 1)$ & Mild-Moderate & $2-4$ & \\
\hline Docetaxel & 1 & 3 & 25 & $0(0 / 1)$ & Moderate & 2 & \\
\hline $\begin{array}{l}\text { Gemcitabine } \\
\text { Cisplatin }\end{array}$ & 1 & 3 & 25 & $0(0 / 1)$ & Moderate & 4 & \\
\hline Enzalutamide & 1 & 3 & 25 & $100(1 / 0)$ & Mild & 3 & \\
\hline Pazopanib & 4 & 14 & 22 & $25(1 / 3)$ & Mild-Moderate & $2-3$ & \\
\hline Abiraterone & 1 & 7 & 12.5 & $100(1 / 0)$ & Mild & 3 & \\
\hline
\end{tabular}

The combination therapy of paclitaxel and carboplatin was associated with the highest DILI frequency (67\%), where 2 of the 3 patients in this study who were exposed to this regimen had DILI. The taxane containing chemotherapeutic regimens showed a DILI frequency of $50 \%$ (5/10). Regimens that included at least one platinum derivative showed a DILI frequency of $38 \%(5 / 13)$, and $60 \%$ of these cases were in bladder cancer. Pazopanib treatment had a DILI frequency of $22 \%$, where 4 out of 18 patients taking this drug had an episode of DILI. DILI latency from pazopanib treatment initiation ranged from 27 to 89 days, with a median of 42 days. Chemotherapy drugs or regimens without any associated cases of DILI included the following: bicalutamide $(n=8)$, firmagon $(n=3)$, and MVAC (methotrexate, vinblastine, doxorubicin and cisplatin) $(n=2)$.

\section{Discussion}

DILI and its effect on chemotherapy treatment have historically been underreported. We evaluated the cumulative incidence of DILI and its clinical significance in patients undergoing chemotherapy for the treatment of prostate, bladder, testicular, and renal cancers during a two-year period. This study, to our knowledge is the first combined report on DILI in patients with the aforementioned GU malignancies.

The cumulative incidence of DILI in our study cohort was $6.1 \%$, which increased to $18.9 \%$ if those without known normal baseline or follow-up LT were excluded. The reported incidence of DILI in patients with cancer has been widely variable, from $1-31 \%$ in patients with lung, breast, and gastrointestinal malignancies $[10,11]$. In part this is due to the use of different definitions of DILI used in these studies. Our definition of DILI was slightly less stringent than that employed 
by US DILIN since subjects were followed prospectively and had protocol driven liver biochemistries drawn. Even so, 33\% percent did meet US DILIN criteria for ALT ( $>5 x$ ULN) [8]. The DILI episodes were clinically significant as the majority of patients (10/17) experienced a change in their treatment plans. No significant difference in mortality was identified between DILI cases and controls, but the size of the cohort was relatively small, and median follow up time was perhaps not long enough. Mortality data in patients with DILI during chemotherapy are limited to perioperative mortality in patients who experienced liver injury from chemotherapy before undergoing surgical resection of liver metastases $[11,12]$.

The only established treatment for DILI is $\mathrm{N}$-acetylcysteine for acetaminophen toxicity, and treatment of DILI caused by other agents has only been evaluated in small scale studies. The latter studies show some benefit to administering prophylactic compounds to prevent DILI in patients undergoing chemotherapy [13,14]. Agents investigated included bicyclol and tiopronin. However, the cohort sizes were small and limited to specific chemotherapeutic agents, therefore, decreasing the generalizability of the results to the wider oncology population.

We compared DILI frequencies from different chemotherapeutic agents and found a surprisingly high frequency for certain drugs and/or drug classes. Chemotherapy containing taxane and platinum-based compounds showed a DILI frequency of $50 \%$ and $38 \%$ respectively. In addition, the combination of paclitaxel and carboplatin was associated with DILI in $2 / 3$ patients. Though the sample size is too small for these numbers to be applicable to all chemotherapy patients, for multiple chemotherapeutic agents this is the first real world report on DILI frequencies, outside of clinical trials. The platinum-based drugs in this study were cisplatin and carboplatin which, according to the FDA warnings can cause aspartate aminotransferase (AST) elevations in $16-23 \%$ of patients being treated for ovarian cancer. However, the degree of elevation in AST was not reported. Docetaxel monotherapy associated DILI, for example, showed an incidence of $25 \%$ in our study, which is higher than what has been previously reported in a study which showed an incidence of $10 \%$ in patients with metastatic breast cancer [15]. The combination of gemcitabine and cisplatin showed a DILI frequency of $25 \%$ with grade 4 toxicity according to the CTCAE severity scale. The FDA label reports the combination of gemcitabine and cisplatin to be associated with only a $1-2 \%$ incidence of grade 3 or 4 liver toxicity $[16,17]$. Our results suggest the frequency of DILI for a given drug or drug combination may vary based on the cancer being treated.

Pazopanib, a multi targeted tyrosine kinase inhibitor used to treat metastatic renal cell carcinoma and sarcomas, showed a cumulative DILI incidence of $22 \%$. It is a drug that has a wellestablished effect of hepatotoxicity. Meta-analysis on the effect of pazopanib showed an incidence of $20 \%$ for hepatocellular DILI as defined as ALT>3x ULN and $6 \%$ for cholestatic DILI as defined by TB $\geq 2 x$ ULN [7]. Conversely, in our study, 4 patients experienced DILI from pazopanib, 1 with hepatocellular and 3 with cholestatic patterns of liver injury. The inclusion criteria for the studies mentioned in this metaanalysis were less stringent than ours as they accepted baseline ALT<2.5 ULN, making it more likely that patients with baseline abnormal liver chemistries from other causes were included. In the meta-analysis cases the proportion of different cancers varied and elimination of alternative etiologies for the elevations in LT results was less extensive than in our study. For example, $11 \%$ of cases in the meta-analysis did not have normalization of their ALT, which was eventually attributed to end organ damage from cancer progression. The meta-analysis reveals a median latency to DILI of 42 days, which did mirror our finding. The mechanism of liver injury by pazopanib remains unclear as there is no reported case that was severe enough to warrant a liver biopsy.

Our results indicate that age, gender, $\mathrm{BMI}$, race, and ethnic group were not predictive of the occurrence of DILI from pazopanib. A meta-analysis of pazopanib monotherapy in cancer clinical trials by Powles et al. reported that pazopanib associated DILI was associated with older age $\geq 60$ years, previous cancer directed therapy, and baseline ALT abnormalities [7]. Our subject number was much smaller than in this meta-analysis, which made our detection of these associations less likely and we excluded those with baseline ALT abnormalities to limit the influence of confounding factors. DILI in those with underlying liver disease or injury is not well studied. The inverse association we detected of baseline serum albumin with DILI from pazopanib as well as nonpazopanib cases has not been reported previously. For drugs normally highly bound by albumin, such as pazopanib, the unbound drug fraction would increase as albumin levels decrease. Whether, the unbound fraction of pazopanib plays a role in its hepatotoxicity is unknown [18]. Another explanation is that lower albumin is an indication of poor baseline liver function, which may lead to a higher susceptibility to DILI. As noted above, it is unclear how poor baseline liver function may affect DILI frequency.

As shown in Table 5, all the cases of DILI were mild to moderate in severity. Though DILI is historically a major cause of drug withdrawal from the market [3], the clinical benefits of these drugs are believed to outweigh their risks. Our results do not indicate that action is warranted to halt the usage of these drugs for those with life-threatening malignancies. Unlike older chemotherapy agents, the newer biologic agents being using for cancer treatment may cause immune-mediated liver injury similar to autoimmune hepatitis [19]. Our results are a clear reminder that appropriate monitoring is needed to minimize hepatic side effects from this new class of cancer drugs.

\section{Conclusion}

In conclusion, we report a cumulative incidence of DILI of $6.1 \%$ in patients undergoing treatment for $\mathrm{GU}$ malignancies and highly frequent (59\%) changes in treatment plans as a result of DILI, which is not optimal for cancer management and perhaps patient outcomes. Thus, DILI has the potential to significantly affect both immediate survival and long-term 
survival in patients with cancer. Prospective studies in those undergoing chemotherapy are needed to identify additional pre-treatment risk factors for DILI, and to evaluate prophylactic agents to prevent chemotherapy associated DILI.

\section{Acknowledgement}

This work was supported by U01 DK100928 grant.

\section{References}

1. Navarro VJ, Barnhart H, Bonkovsky HL, Davern T, Fontana RJ, et al. (2014) Liver injury from herbals and dietary supplements in the US Drug-Induced Liver Injury Network. Hepatology 60: 1399-1408.

2. Andrade RJ, Lucena MI, Fernández MC, Pelaez G, Pachkoria K, et al. (2005) Drug-induced liver injury: an analysis of 461 incidences submitted to the Spanish registry over a 10-year period. Gastroenterology 129: 512-521.

3. Navarro VJ, Senior JR (2006) Drug related hepatotoxicity. N Engl J Med 354: 731-739.

4. Reuben A, Koch DG, Lee WM (2010) Drug-induced acute liver failure: results of a US multicenter, prospective study. Hepatology 52: 2065-2076.

5. Kleiner DE, Chalasani NP, Lee WM, Fontana RJ, Bonkovsky HL, et al. (2014) Hepatic histological findings in suspected druginduced liver injury: systematic evaluation and clinical associations. Hepatology 59: 661-670.

6. Bahirwani R, Reddy KR (2014) Drug-induced liver injury due to cancer chemotherapeutic agents. Seminars in liver Dis 34: $162-171$

7. Powles T, Bracarda S, Chen M, Norry E, Compton N, et al. (2015) Characterisation of liver chemistry abnormalities associated with pazopanib monotherapy: A systematic review and metaanalysis of clinical trials in advanced cancer patients. Eur J Cancer 51: 1293-1302.

8. Fontana RJ, Watkins PB, Bonkovsky HL, Chalasani N, Davern T, et al. (2009) Drug-induced liver injury network (DILIN) prospective study. Drug Saf 32: 55-68.
9. https://ctep.cancer.gov/protocolDevelopment/ electronic_applications/ctc.htm

10. Ulcickas Yood M, Bortolini M, Casso D, Beck JG, Oliveria SA, et al. (2015) Incidence of liver injury among cancer patients receiving chemotherapy in an integrated health system. Pharmacoepidemiology Drug Saf 24: 427-434.

11. Vauthey JN, Pawlik TM, Ribero D, Wu TT, Zorzi D, et al. (2006) Chemotherapy regimen predicts steatohepatitis and an increase in 90-day mortality after surgery for hepatic colorectal metastases. J Clin Oncol 24: 2065-2072.

12. Pawlik TM, Olino K, Gleisner AL, Torbenson $M$, Schulick $R$, et al. (2007) Preoperative chemotherapy for colorectal liver metastases: impact on hepatic histology and postoperative outcome. J Gastrointest Surg 11: 860-868.

13. Li X, Zhou J, Chen S, Guan M, Wang Y, et al. (2014) Role of bicyclol in preventing chemotherapeutic agent-induced liver injury in patients over 60 years of age with cancer. J Int Med Res 42: 906-14.

14. Li XP, Wen F, Yang W, Deng YB, Li M, et al. (2014) The role of tiopronin for the prevention of chemotherapy-related liver toxicity in advanced colorectal cancer patients treated with mFOLFOX7: A prospective analysis. Tumori J 100: 446-451.

15. Wang Z, Liang X, Yu J, Zheng X, Zhu Y, et al. (2012) Non-genetic risk factors and predicting efficacy for docetaxel-drug-induced liver injury among metastatic breast cancer patients. J Gastroenterol Hepatol 27: 1348-1352.

16. https://www.accessdata.fda.gov/drugsatfda_docs/label/ 2004/020509s029lbl.pdf

17. https://www.accessdata.fda.gov/drugsatfda_docs/label/ 2011/018057s080lbl.pdf

18. Imbs DC, Paludetto $M N$, Négrier $S$, Powell $H$, Lafont $T$, et al. (2016) Determination of unbound fraction of pazopanib in vitro and in cancer patients reveals albumin as the main binding site. Invest New Drugs 34: 41-48.

19. Suzman DL, Pelosof L, Rosenberg A, Avigan MI (2018) Hepatotoxicity of immune checkpoint inhibitors: An evolving picture of risk associated with a vital class of immunotherapy agents. Liver Int 38: 976-987. 